\title{
The Comparison between one and two dimensional up-and-down Method
}

\author{
Huan Xue ${ }^{1, a}$, Yuxi Ma ${ }^{1}$, Rui Ge $^{1}$, Long Yan ${ }^{1}$, Lanxiang Kuang ${ }^{1}$ \\ ${ }^{1}$ Research and Development Center of Wuhan Iron and Steel group Corp., Wuhan, 430080, China \\ astonemechanics@163.com
}

Keywords: WL700, Two dimensional up-and down Method, Fatigue Limit Strength, Relative Derivation.

\begin{abstract}
The fatigue performances of two types of the automotive frame steel (AFS) include WL700 and WL510 are investigated. In order to take the thickness effect into account, two series of WL510 samples with $6 \mathrm{~mm}$ and 8mm thickness respectively are selected. The fatigue strengths are calculated by one and two dimensional up-and-down method under dfifferent probability of failure and believe. The result shows that the relative deviation of fatigue strength using one dimensional method is much 3 to 4 times larger than two dimensional method. In addition, more experienced data brings more accurate result. The relative deviation of fatigue strengths become larger, when the probability of failure increased and probability of believe decreased.
\end{abstract}

\section{Introduction}

W. J. Dixon and A. M. Mood firstly proposed the up-and-down method for sensitivity testing, which is widely used in fatigue testing and developed by many other experts [1-3]. However, the estimates for variance are not exact and the previous data can not be effectively used in the traditional up-and-down method which leads low test efficiency. The fatigue test requirement in many laboratory increases rapidly, along with the rapid development of the rail, automobile and other new steel products. In order to solve the contradiction, many researchers try to develop new fatigue testing method with higher efficiency [4,5]. The most recommended one is the two dimensional up-and-down method [6]. The meaning of the two dimensional is that this method takes both the current data and the previous data into account. As the information used in two dimensional method (TDM) is much larger and more abundant than the traditional one dimensional method (ODM) which only cares about the current data [7]. Therefore, the TDM can save many specimens and testing time under the same accuracy requirement. In the other aspect, it can enhance accuracy of the test result with the same number of specimens compares with the traditional one.

In this study, the fatigue performances of two types of the automotive frame steel (AFS) include WL700 and WL510 are investigated. In order to take the thickness effect into account, two series of WL510 samples with $6 \mathrm{~mm}$ and $8 \mathrm{~mm}$ thickness respectively are selected. The important parameters of the two dimensional up-and-down method is introduced in the next section. The fatigue test results using traditional method are analyzed in Section 3. Two cases of testing using TDM are compared with the tradition one in Section 4. Some important conclusions are made in the final section.

\section{The two dimensional up-and-down method}

When the probability of believe of percentages is at least $\gamma$, the one side confidence low limits $x_{P L}$ and one side confidence high limits $x_{P U}$ can be given as [8]

$$
x_{P L}=\hat{\mu}-\sqrt{2} k_{P} s \quad x_{P U}=\hat{\mu}+\sqrt{2} k_{1-P} s
$$

Where $k_{p}$ and $k_{1-p}$ is the two dimensional one side confidence coefficient when probability of believe is $\gamma$. If $\mathrm{n}>3 k_{p}$ can be written as

$$
k_{P}=\sqrt{\frac{2 n-3}{2 n-4}}\left(1-\frac{u_{r}^{2}}{w}\right)^{-1}\left[-u_{p}+u_{r} \sqrt{\frac{1}{2 n}\left(1-\frac{u_{r}^{2}}{w}\right)+\frac{u_{P}^{2}}{w}}\right] \quad w=2\left(n+u_{r}-c-1-1 / \sqrt{n+u_{r}-c-1}\right)
$$

$\mathrm{c}$ is the function of $\mathrm{P}$ and $\gamma$, when $\mathrm{n}>6$, c can be set as a constant value 0.64. 
The notable difference between TDM and ODM is that the TDM takes the previous information into account. The combined variance is one of the most important parameter of the TDM which connects the previous and current data. We set there are $\mathrm{m}$ series of previous data and one series of current data form ODM. The average value, variance of previous data are $\mu_{i}, s_{i}^{2}$ respectively. Their degree of freedom are $v_{i}, \mathrm{i}=1,2,3 \ldots \ldots \mathrm{m}$, which are independent from others. The average value and variance of current data are $\mu$ and $s^{2}$, while the degree of freedom is $n-1$. For the two dimensional consideration, the whole data degree of freedom is $v+n-1$, where $v=\sum_{i=1}^{m} v_{i}$. According to Ref [7], the combined variance is

$$
s_{c}^{2}=\frac{\sum_{i=1}^{m} v_{i} s_{i}^{2}+(n-1) s^{2}}{v+n-1}
$$

For fatigue limit test, when $k_{p}>0$, if the dispersibility of previous data is larger than the current data with $\mathrm{E}\left(s_{c}^{2}\right) \geqslant \mathrm{E}\left(\mathrm{s}^{2}\right)$, the one side low limit of confidence $\mathrm{x}_{\mathrm{PL}}$ of sensitive variable $x$ with percentiles $x_{p}$ can be written as

$$
\begin{gathered}
x_{P L}=\hat{\mu}-\sqrt{2} k_{P} s_{c} \\
k_{P}=\sqrt{\frac{2 v+2 n-3}{2 v+2 n-4}}\left(1-\frac{u_{r}^{2}}{w}\right)^{-1}\left[-u_{p}+u_{r} \sqrt{\frac{1}{2 n}\left(1-\frac{u_{r}^{2}}{w}\right)+\frac{u_{P}^{2}}{w}}\right] \\
w=2\left(v+n+u_{r}-c-1-1 / \sqrt{v+n+u_{r}-c-1}\right)
\end{gathered}
$$

\begin{tabular}{|c|c|c|c|c|c|c|c|c|c|c|c|c|c|c|c|c|c|c|}
\hline \multirow{2}{*}{$\begin{array}{c}\mathrm{S}_{\mathrm{a}} \\
(\mathrm{Mpa})\end{array}$} & \multicolumn{18}{|c|}{ Test Sequence } \\
\hline & 1 & 2 & 3 & 4 & 5 & 6 & 7 & 8 & 9 & 10 & 11 & 12 & 13 & 14 & 15 & 16 & 17 & 18 \\
\hline 246.5 & & & & & & & & & $\mathrm{X}$ & & & & & & & & & \\
\hline 238 & & $\mathrm{X}$ & & & & & & Pass & & $\mathrm{X}$ & & & & $\mathrm{X}$ & & & & $\mathrm{X}$ \\
\hline 229.5 & Pass & & $\mathrm{X}$ & & $\mathrm{X}$ & & Pass & & & & $\mathrm{X}$ & & Pass & & $\mathrm{X}$ & & Pass & \\
\hline 221 & & & & Pass & & Pass & & & & & & Pass & & & & Pass & & \\
\hline
\end{tabular}

According to Ref [7], up=-1.282, ur=1.645, c=0.655.

\section{Fatigue Experiment on WL700}

Table 1 Up and down fatigue test result of WL700

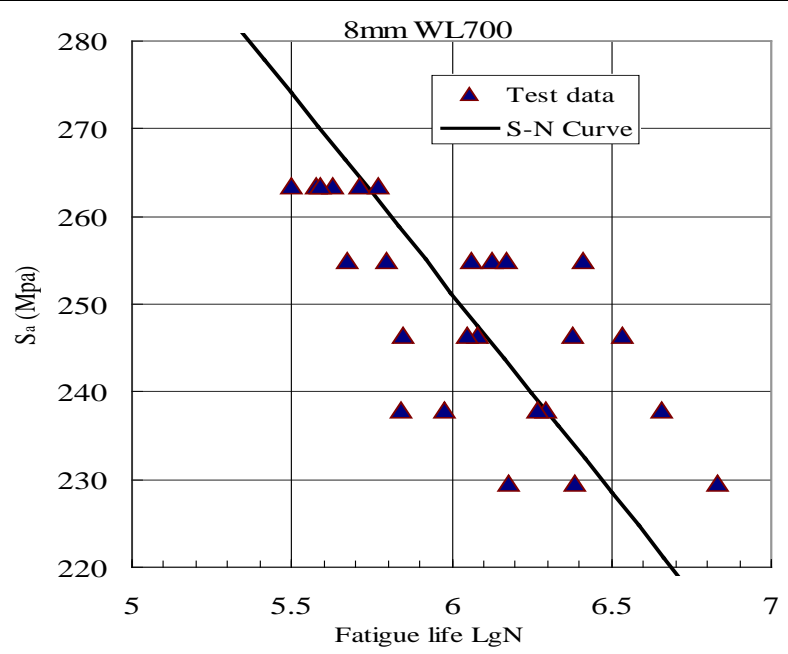

Figure 1 S-N curve of WL700

The test equipment is high frequency fatigue tester PLG-100. 40 fatigue test samples of WL700 are prepared base on Chinese experiment Standard GB/T 3075-2008 and GB/T24176-2009 [9, 10]. In order to reduce the initial flaw and crack source produced by machining factor, the samples are manufactured by wire-electrode cutting and the side arc surface are polished. The tensile-tensile 
forces are applied in the test with cycling stress ratio $\mathrm{R}=0.15$. The tests are under room temperature. The up and down fatigue test result is shown in Table 1. The testing should follow the two rules stated below: a. "Pass": Each time a test sample survives $10^{7}$ cycles, the load must be increased with 8.5 MPa for the next test with a quite new test sample; b. " $X$ ”: If the test sample fails before $10^{7}$ cycles, the load must be decreased with $8.5 \mathrm{MPa}$ for the next randomly chosen test sample. The S-N curve is shown in Figure 1.

\section{Experience Fatigue data on WL510}

Table 2 Up and down fatigue test result of WL510 with 6mm thickness

\begin{tabular}{|c|c|c|c|c|c|c|c|c|c|c|}
\hline $\mathrm{S}_{\mathrm{a}}$ & \multicolumn{10}{|c|}{ Test Sequence } \\
\cline { 2 - 11 }$(\mathrm{Mpa})$ & 1 & 2 & 3 & 4 & 5 & 6 & 7 & 8 & 9 & 10 \\
\hline 204 & & & & & $\mathrm{X}$ & & $\mathrm{X}$ & & & \\
\hline 196 & & $\mathrm{X}$ & & Pass & & Pass & & $\mathrm{X}$ & & $\mathrm{X}$ \\
\hline 187 & Pass & & Pass & & & & & & Pass & \\
\hline
\end{tabular}

Table 3 Up and down fatigue test result of WL510 with 8mm thickness

\begin{tabular}{|c|c|c|c|c|c|c|c|c|c|c|}
\hline $\mathrm{S}_{\mathrm{a}}$ & \multicolumn{9}{|c|}{ Test Sequence } \\
\cline { 2 - 11 }$(\mathrm{Mpa})$ & 1 & 2 & 3 & 4 & 5 & 6 & 7 & 8 & 9 & 10 \\
\hline 196 & & & $\mathrm{X}$ & & & & & & $\mathrm{X}$ & \\
\hline 187 & & Pass & & $\mathrm{X}$ & & $\mathrm{X}$ & & Pass & & $\mathrm{X}$ \\
\hline 179 & Pass & & & & Pass & & Pass & & & \\
\hline
\end{tabular}

10 samples of $6 \mathrm{~mm}$ WL510, 8mm WL 510 were tested before respectively. The two groups of samples have the same size and shape on cross-section with WL700. The test stress steps are 8.5 and 11.0 Mpa on WL510 respectively. The mean fatigue strength, fatigue limit and deviation are 194.7, 165.9, 6.3 and 186.7, 157.9, 6.3 Mpa respectively. It should be note that for the same materials the fatigue strength of thicker specimen is lower than the thin one. The possible reasons are the thicker specimen may have more initial crack and inside inclusions.

Table 4 Comparison between one and two dimensional up-and-down Method

\begin{tabular}{|c|c|c|c|c|c|c|c|c|}
\hline \multirow{2}{*}{$\begin{array}{l}\text { Sample } \\
\text { No. }\end{array}$} & \multirow{2}{*}{$\begin{array}{l}\text { probability } \\
\text { of failure }\end{array}$} & \multirow{2}{*}{$\begin{array}{l}\text { probability } \\
\text { of believe }\end{array}$} & \multicolumn{3}{|c|}{ Fatigue strength } & \multicolumn{3}{|c|}{ Relative deviation } \\
\hline & & & $\begin{array}{l}\text { ODM } \\
\text { (Mpa) }\end{array}$ & $\begin{array}{l}\text { TDM } 1 \\
\text { (Mpa) }\end{array}$ & $\begin{array}{l}\text { TDM } 2 \\
\text { (Mpa) }\end{array}$ & $\begin{array}{c}\text { ODM } \\
(\%)\end{array}$ & $\begin{array}{c}\text { TDM } 1 \\
(\%)\end{array}$ & $\begin{array}{c}\text { TDM } 2 \\
(\%)\end{array}$ \\
\hline 10 & $90 \%$ & \multirow[t]{5}{*}{$10 \%$} & 202.1 & \begin{tabular}{|l|}
206.3 \\
\end{tabular} & 207.7 & 4.1 & 2.2 & 1.5 \\
\hline 12 & & & 204.1 & 207.1 & 208.3 & 3.2 & 1.8 & 1.2 \\
\hline 14 & & & 207.4 & 209.4 & 210.3 & 1.6 & 0.7 & 0.3 \\
\hline 16 & & & 208.0 & 209.6 & 211.3 & 1.3 & 0.6 & 0.2 \\
\hline 18 & & & 209.9 & 211.0 & 211.6 & 1 & 1 & 1 \\
\hline 10 & $95 \%$ & \multirow[t]{5}{*}{$10 \%$} & 195.1 & 200.3 & 202.0 & 6.2 & 3.7 & 2.9 \\
\hline 12 & & & 198.8 & 202.4 & 203.8 & 4.4 & 2.7 & 2.0 \\
\hline 14 & & & 203.3 & 205.6 & 206.6 & 2.2 & 1.1 & 0.7 \\
\hline 16 & & & 204.5 & 206.3 & 207.2 & 1.7 & 0.8 & 0.4 \\
\hline 18 & & & 206.9 & 208.2 & 208.8 & 1 & 1 & 1 \\
\hline 10 & $95 \%$ & \multirow[t]{5}{*}{$1 \%$} & 167.8 & 176.8 & 179.7 & 12.1 & 7.4 & 5.9 \\
\hline 12 & & & 175.1 & 181.4 & 183.8 & 8.3 & 5.0 & 3.8 \\
\hline 14 & & & 182.6 & 186.6 & 188.3 & 4.4 & 2.3 & 1.4 \\
\hline 16 & & & 185.2 & 188.4 & 189.9 & 3.0 & 1.3 & 0.6 \\
\hline 18 & & & 189.1 & \begin{tabular}{|l}
191.3 \\
\end{tabular} & 192.5 & 1 & 1 & 1 \\
\hline
\end{tabular}

\section{Results comparison}

The fatigue strengths are calculated by one and two dimensional up-and-down method under fifferent probability of failure and believe. The results are shown and compared in Table 4. The probability of failure and probability of believe are set to be $0.9,0.95,0.95$ and $0.1,0.1,0.01$ respectively in three cases. In each case, 10 to 18 tested samples are taken into account. The two dimensional up-and-down method only considers the 6mm WL510 experienced data is named to be 
TDM 1, and the method uses both $6 \mathrm{~mm}$ and 8mm WL510 experienced data is named to be TDM 2 . The average value of fatigue strengths using three method with 18 tested samples are set to be the standard value. It can be found that the relative deviation of fatigue strength using TDM is much smaller than ODM. The more tested samples are selected, the closer of the calculated fatigue strength to the standard one. In another aspect, the result uses TDM 2 is closer to the standard value than TDM 1, which means more experienced data brings more accurate result. In both three cases, the fatigue strength deviation of ODM is nearly 3 to 4 times larger than TDM 2. In addition, when the probability of failure increased and probability of believe decreased, the relative deviation of fatigue strengths become larger.

\section{Conclusions}

(1) The fatigue performances of two types of automotive frame steel include WL510 and WL700 are tested with both one and two up and down dimensional method.

(2) The relative deviation of fatigue strength using TDM is much smaller than ODM. In both three cases, the fatigue strength deviation of ODM is nearly 3 to 4 times larger than TDM 2.

(3) More experienced data brings more accurate result.

(4) The relative deviation of fatigue strengths become larger when the probability of failure increased and probability of believe decreased.

\section{References}

[1] Z. T. Gao, X. T. Jiang, J. J. Xiong: Fatigue performance experimental design and data processing, Press of Beijing University of Aeronautics and Astronautics.

[2] C. R. Chen: Fatigue and fracture, Press of Huazhong university of science and technology, 2002

[3] W. J. Dixon, A. M. Mood. A Method for obtaining and analyzing sensitivity data. J Amer Statistical Assoc, 1928, 43, 109-126

[4] J. Y. Zhang, D. L. Wang. Up and down method for fatigue strength with two details in series. Journal of Experimental Mechanics, 2006, 21(5), 579-583

[5] Lin S K, Lee Y L, Lu M W. Evaluation of the staircase and the accelerated test methods for fatigue limit distributions[J].International Journal of Fatigue, 2001, 23(1), 75 83

[6] H. M. Fu, G. Yin, Two-dimensional up-and-down method. Acta Aeronautica Et Astronautica Sinica 1998, 19 (6), 748-753

[7] H. M. Fu, G. Yin, A method for determining fatigue limits with small samples. Journal of Mechanical Strength, 1999, 21(2), 115-117

[8] H. M. Fu, Formulas for tolerance limits and confidence limits of nomal population percentiles and percentages. Acta Aeronautica Et Astronautica Sinica 1994, 15 (1), 94-101

[9] Chinese National Strandard: Metallic materials Fatigue testing- Axial force controlled method, GB/T3075-2008.

[10] Chinese National Strandard: Metallic materials Fatigue testing Statistical planning and analysis of data, GB/T24176-2009. 